Pacific Journal of Mathematics

CERTAIN SUBVARIETIES OF A WEAKLY PSEUDOCONVEX

K. ADACHI 


\title{
EXTENDING BOUNDED HOLOMORPHIC FUNCTIONS FROM CERTAIN SUBVARIETIES OF A WEAKLY PSEUDOCONVEX DOMAIN
}

\author{
KenZō ADACHI
}

Let $D$ be a weakly pseudoconvex domain in $C^{n}$ with $C^{\infty}$-boundary and $\Delta$ be a hypersurface in $D$ which intersects $\partial D$ transversally. If $\partial \Delta$ consists of strictly pseudoconvex boundary points of $D$, then any bounded holomorphic function in $\Delta$ can be extended to a bounded holomorphic function in $D$.

1. Introduction. G. M. Henkin [5] proved that any bounded holomorphic function defined on an analytic closed submanifold in general position in a strongly pseudoconvex domain can be continued to a bounded holomorphic function in the entire domain. The related results have been given by the author [1] and J. E. Fornaess [4]. In this paper, we extend this problem to the weakly pseudoconvex case. Our proof depends on the integral formula constructed by E. L. Stout [8], and the kernel function constructed by F. Beatrous, Jr. [3] which was used to obtain a Hölder estimate for solutions to $\bar{\partial}$-problem in weakly pseudoconvex domains.

2. Let $\Omega$ be a bounded domain in $C^{N+1}$ with $C^{\infty}$-boundary. We shall denote by $O(\Omega)$ the space of holomorphic functions in $\Omega$. We shall also denote by $H^{\infty}(\Omega)$ the space of bounded holomorphic functions on $\Omega$ and by $A(\Omega)$ the subspace of $H^{\infty}(\Omega)$ of functions which extend continuously to $\bar{\Omega}$.

Definition 1. (R. M. Range [7]) A point $\lambda \in \partial \Omega$ is a strictly pseudoconvex boundary point if there are a neighborhood $U$ of $\lambda$ and a $C^{\infty}$ function $\phi: U \rightarrow R$ such that:

(a) $U \cap \Omega=\{z \in U: \phi(z)<0\}$;

(b) $\Sigma\left(\partial^{2} \phi(\lambda) / \partial z_{i} \partial \bar{z}_{j}\right) w_{i} \bar{w}_{j}>0$ for all $w \in C^{N+1}-(0)$;

(c) $d \phi(\lambda) \neq 0$.

The set of strictly pseudoconvex boundary points of $\Omega$ will be denoted by $S(\Omega)$. It follows from Definition 1 that $S(\Omega)$ is an open subset of the boundary $\partial \Omega$. 
Let $D$ be a pseudoconvex domain in $C^{N+1}$ with $C^{\infty}$-boundary. We fix a function $F \in O(\bar{D}), F \neq 0$. Then $F$ is holomorphic in a domain $\tilde{D}$ with $\bar{D} \subset \tilde{D}$. We set $\tilde{\Delta}=\{z \in \tilde{D}: F(z)=0\}$ and $\Delta=\tilde{\Delta} \cap D$. We make the following assumptions:

(a) $\Delta$ is a non-empty connected set;

(b) $d F \neq 0$ on $\partial \Delta$;

(c) $\tilde{\Delta}$ meets $\partial D$ transversally;

(d) $\partial \Delta \subset S(D)$.

In this setting, we have the following:

THEOREM. Under hypotheses (a)-(d), there exists a continuous linear extension operator $L: H^{\infty}(\Delta) \rightarrow H^{\infty}(D)$. Moreover if $\Delta$ has no singular points then $L(A(\Delta)) \subset A(D)$.

In order to prove this theorem, we use the function $\Phi(\zeta, z)$ in the following proposition which was constructed by F. Beatrous, Jr. ([3], Theorem 2.1).

Proposition 1. Let $k$ be a positive integer $(k \geq 3)$. There are $a$ neighborhood $U$ of $\partial \Delta$, a smooth positive function $r$ on $U$, and a $C^{k}$ function $\Phi$ on $U \times \bar{D}$ with the following properties:

(i) For each $\zeta \in U, \Phi(\zeta, \cdot) \in C^{k}(\bar{D}) \cap O(D)$.

(ii) $G(\zeta, z)=\Phi(\zeta, z) / T(\zeta, z)$ is a non-vanishing $C^{k}$ function on $\{(\zeta, z) \in U \times \bar{D}:|\zeta-z| \leq r(z)\}$.

(iii) $\Phi(\zeta, z) \neq 0$ if $|\zeta-z| \geq r(z)$.

(iv) $\operatorname{Re} T(\zeta, z)>\rho(\zeta)-\rho(z)+r(z)|\zeta-z|^{2}$ if $|\zeta-z| \leq r(\zeta)$, where $\rho$ is the defining function for the domain $D$ constructed by $F$. Beatrous, $J r$., and

$$
T(\zeta, z)=-2 \sum_{i} \frac{\partial \rho}{\partial z_{i}}(\zeta)\left(z_{i}-\zeta_{l}\right)-\sum_{\imath, J} \frac{\partial^{2} \rho}{\partial z_{i} \partial z_{l}}(\zeta)\left(z_{l}-\zeta_{l}\right)\left(z_{l}-\zeta_{j}\right)
$$

Moreover we can extend $\Phi(\zeta, z)$ to a $C^{k}$ function on a neighborhood of $\partial D \times \bar{D}$, holomorphic in $z$ such that $\Phi(\zeta, z)$ satisfies $\Phi(\zeta, z)=$ $\sum_{J=1}^{N+1} P_{J}(\zeta, z)\left(\zeta,-z_{j}\right)$, and $\Phi(\zeta, z) \neq 0$ if $\rho(\zeta)>\rho(z)$, where $P_{J}(\zeta, z)$ is a $C^{k}$ function on a neighborhood of $\partial D \times \bar{D}$, holomorphic in $z$.

Let $D_{v}=\left\{z \in D: \rho(z)<-\varepsilon_{\nu}\right\}$ and $\Delta_{\nu}=\Delta \cap D_{\nu}$, where $\left\{\varepsilon_{v}\right\}$ is a sequence of sufficiently small strictly decreasing positive numbers converging to 0. By E. L. Stout [8], we have the following: 
Proposition 2. If $f \in H^{\infty}(\Delta)$, then the following formula holds for all $z \in \Delta_{\nu}$ and all sufficiently large $\nu$ :

$$
f(z)=\int_{\partial \Delta_{\nu}} f(\zeta) \frac{\tilde{\Psi}(\zeta, z) \tilde{\omega}_{F}}{\Phi(\zeta, z)^{N}\|\operatorname{grad} F(\zeta)\|}=\int_{\partial \Delta_{\nu}} \frac{f(\zeta) K(\zeta, z)}{\Phi(\zeta, z)^{N}},
$$

where $\tilde{\Psi}(\zeta, z)$ is a $C^{k-1}(0, N-1)$ form in a neighborhood of $\partial D \times \bar{D}$ and, for each $\zeta$ near $\partial D$, coefficients of $\tilde{\Psi}(\zeta, \cdot)$ are holomorphic in $D$. One could arrange for $\tilde{\Psi}(\zeta, \cdot)$ to be holomorphic on $\bar{D}$ if $\bar{D}$ were assumed to have a pseudoconvex neighborhood basis. $\tilde{\omega}_{F}$ is given by

$$
\tilde{\omega}_{F}=\sum_{j=1}^{N+1}(-1)^{j-1} \bar{F}_{j} d \zeta_{1} \wedge \cdots \wedge \widehat{d \zeta_{j}} \wedge \cdots \wedge d \zeta_{N+1},
$$

where $F_{j}=\partial F / \partial z_{j}, j=1, \ldots, N+1$, and $\wedge$ means the symbol is to be omitted. Therefore $K(\zeta, z)$ is a $C^{k-1}(N, N-1)$ form on a neighborhood of $\partial D \times \bar{D}$ and for each $\zeta$ near $\partial D$, coefficients of $K(\zeta, \cdot)$ are holomorphic in D.

We set

$$
H_{\nu}(z)=\int_{\partial \Delta_{\nu}} \frac{f(\zeta) K(\zeta, z)}{\Phi(\zeta, z)^{N}} \quad \text { for } z \in \bar{D}_{\nu} \mid \partial \Delta_{\nu}
$$

and

$$
L(f)(z)=H(z)=\lim _{\nu \rightarrow \infty} H_{\nu}(z) \quad \text { for } z \in \bar{D} \mid \partial \Delta
$$

LEMMA 1. $H(z)$ is holomorphic on $D$ and $H(z)=f(z)$ for all $z \in \Delta$.

Proof. For $z \in W \Subset D_{\nu_{0}}, \nu>\mu \geq \nu_{0}$, we have

$$
H_{\nu}(z)-H_{\mu}(z)=\int_{\partial\left(\Delta_{\nu}-\Delta_{\mu}\right)} \frac{f(\zeta) K(\zeta, z)}{\Phi(\zeta, z)^{N}}=\int_{\Delta_{\nu}-\Delta_{\mu}} f(\zeta) \bar{\partial}_{\zeta}\left(\frac{K(\zeta, z)}{\Phi(\zeta, z)^{N}}\right) .
$$

Since the form $\bar{\partial}_{\zeta}\left(K(\zeta, z) / \Phi(\zeta, z)^{N}\right)$ is bounded for $\zeta \in \Delta_{\nu}-\Delta_{\mu}$ and $z \in W$, there exists a constant $K$ such that

$$
\left|H_{\nu}(z)-H_{\mu}(z)\right| \leq K \sup _{\zeta \in \Delta}|f(\zeta)| \operatorname{Vol}\left(\Delta_{\nu}-\Delta_{\mu}\right) .
$$

Hence $H_{\nu}(z)$ converges locally uniformly on $D$. Therefore $H(z)$ is holomorphic in $D$. By Proposition 2, $H(z)=f(z)$ for all $z \in \Delta$. Therefore Lemma 1 is proved.

We want to show that $H(z) \in H^{\infty}(D)$. Let $S_{z^{0, \sigma}}=\left\{z:\left|z-z^{0}\right|<\sigma\right\}$. Let $z^{0} \in \partial \Delta$. Then there exist a constant $\sigma_{1}>0$ and a biholomorphic 
change of coordinates on a neighborhood of $z^{0}$ such that $\rho$ is strictly convex in a neighborhood of $\bar{D} \cap \bar{S}_{z^{0 . \sigma_{1}}}, \Delta \cap S_{z^{0, \sigma_{1}}}=\left\{z \in S_{z^{0, \sigma_{1}}}: z_{N+1}=0\right\}$ and $\partial \rho\left(z^{0}\right) / \partial z_{1} \neq 0$. Let $0<\sigma_{2}<\sigma_{1}$. Let $z \in S_{z^{0, \sigma_{2}}} \cap D_{\nu}$. We write

$$
H_{\nu}(z)=\int_{\partial \Delta_{\nu} \cap S_{z^{0}, \sigma_{1}}} \frac{f(\zeta) K(\zeta, z)}{\Phi(\zeta, z)^{N}}+\int_{\partial \Delta_{\nu} \mid S_{z^{0, \sigma_{1}}}} \frac{f(\zeta) K(\zeta, z)}{\Phi(\zeta, z)^{N}} .
$$

Then

$$
\left|\int_{\partial \Delta_{\nu} \mid S_{z} 0 . \sigma_{1}} \frac{f(\zeta) K(\zeta, z)}{\Phi(\zeta, z)^{N}}\right| \leq \gamma_{1} \sup _{\zeta \in \Delta_{\nu}}|f(\zeta)|
$$

where $\gamma_{1}$ depends only on $D$ and $\Delta$. We set

$$
\tilde{H}_{\nu}(z)=\int_{\partial \Delta_{\nu} \cap S_{z^{0 . o_{1}}}} \frac{f(\zeta) K(\zeta, z)}{\Phi(\zeta, z)^{N}} .
$$

Then it is sufficient to show that $\left|\tilde{H}_{\nu}(z)\right| \leq \gamma_{2} \sup _{\zeta \in \Delta}|f(\zeta)|$, where $\gamma_{2}$ depends only on $D$ and $\Delta$.

We consider the system of equations for $\zeta^{0}=\left(\zeta_{1}^{0}, \ldots, \zeta_{N+1}^{0}\right)$ of the following form for $z \in S_{z^{0 . \sigma_{2}}}$ :

$$
\begin{gathered}
\sum_{i=1}^{N+1} \frac{\partial \rho}{\partial \zeta_{l}}\left(\zeta^{0}\right)\left(\zeta_{i}^{0}-z_{i}\right)=0 \\
\zeta_{i}^{0}=z_{i} \quad(i=2,3, \ldots, N), \\
\zeta_{N+1}^{0}=0
\end{gathered}
$$

Then we have the following lemma which was proved by G. M. Henkin [5]. But we give the proof of E. Amar [2] which is simpler than Henkin's.

LEMMA 2. There exist positive constants $\sigma_{3}\left(<\sigma_{2}\right), \gamma_{3}$ and $\gamma_{4}$, depending only on $D$ and $\Delta$, such that for any $\sigma \leq \sigma_{3}$ and any $z \in S_{z^{0, \sigma / 2}}$ there exists $a$ unique solution $\zeta^{0}=\zeta^{0}(z)$ of system (2) which belongs to the set $S_{z^{0,0}} \cap \tilde{\Delta}$. Here the point $\zeta^{0}=\zeta^{0}(z)$ has the following properties:

$$
\begin{gathered}
\left|z-\zeta^{0}\right|^{2} \leq \frac{1}{\gamma_{3}}\left[\rho(z)-\rho\left(\zeta^{0}\right)\right], \\
\left|z-\zeta^{0}\right|^{2} \geq\left|z_{N+1}\right|^{2} \geq \gamma_{4}\left[\rho(z)-\rho\left(\zeta^{0}\right)\right], \\
\zeta^{0}=z \quad \text { for any } z \in S_{z^{0, \sigma / 2}} \cap \tilde{\Delta} .
\end{gathered}
$$


Proof. From (2), we have

$$
z_{1}=\zeta_{1}-\frac{\left(\partial \rho(\zeta) / \partial z_{N+1}\right) z_{N+1}}{\partial \rho(\zeta) / \partial z_{1}}=\zeta_{1}-a(\zeta) z_{N+1},
$$

where $a(\zeta)$ is $C^{\infty}$ in a neighborhood of $z^{0}$. There exists $\sigma_{3}>0$ such that for any $\zeta \in B\left(z^{0}, \sigma_{3}\right), z \in B\left(z^{0}, \sigma_{3}\right)$ we have $|\nabla a(\zeta)|\left|z_{N+1}\right| \leq \frac{1}{2}$. We set by recurrence that

$$
\begin{aligned}
& \zeta_{1}^{(1)}=z_{1}, \\
& \zeta^{(\jmath)}=\left(\zeta_{1}^{(j)}, z_{2}, \ldots, z_{N}, z_{N+1}^{0}\right), \\
& \zeta_{1}^{(J)}=z_{1}+a\left(\zeta^{(J-1)}\right) z_{N+1} .
\end{aligned}
$$

If $z$ and $\zeta^{(\prime)}$ are in $B\left(z^{0}, \sigma_{3}\right)$, then

$$
\left|\zeta_{l}^{(J)}-\zeta_{l}^{(J-1)}\right|<\left|z_{N+1}\right||\nabla a|\left|\zeta_{l}^{(j-1)}-\zeta_{l}^{(J-2)}\right|<\frac{1}{2}\left|\zeta_{l}^{(J-1)}-\zeta_{l}^{(J-2)}\right| .
$$

Therefore $\zeta^{(/)}$converges. Then $\lim _{\nu \rightarrow \infty} \zeta^{(J)}=\zeta^{0}$ is the soution of (2). The strict convexity of the function $\rho$ and equations (2) imply the inequalities:

(5) $\rho\left(\zeta^{0}\right)-\rho(z)+\gamma_{3}\left|\zeta^{0}-z\right|^{2} \leq 2 \operatorname{Re} \sum_{i=1}^{N+1} \frac{\partial \rho}{\partial \zeta_{l}}\left(\zeta^{0}\right)\left(\zeta_{l}^{0}-z_{l}\right)=0$,

(6) $\rho\left(\zeta^{0}\right)-\rho(z)+\gamma_{4}^{\prime}\left|\zeta^{0}-z\right|^{2} \geq 2 \operatorname{Re} \sum_{i=1}^{N+1} \frac{\partial \rho}{\partial \zeta_{i}}\left(\zeta^{0}\right)\left(\zeta_{i}^{0}-z_{i}\right)=0$,

where $z \in S_{z^{0, \sigma_{3} / 2}}$. From (5) we have (3). From (6) we have

$$
\left|\zeta^{0}-z\right|^{2} \geq \frac{1}{\gamma_{4}^{\prime}}\left[\rho(z)-\rho\left(\zeta^{0}\right)\right] \text {. }
$$

But

$$
\left|\zeta^{0}-z\right|^{2} \geq\left|z_{N+1}\right|^{2}+\left|\zeta_{1}^{0}-z_{1}\right|^{2} \leq \gamma_{4}^{\prime \prime}\left|z_{N+1}\right|^{2} .
$$

Therefore we have $\left|z_{N+1}\right|^{2} \geq\left(1 / \gamma_{4}\right)\left[\rho(z)-\rho\left(\zeta^{0}\right)\right]$. Therefore Lemma 2 is proved.

For any $z \in \overline{D_{\nu}} \cap S_{z^{0 . \sigma_{2}}} \mid \partial \Delta_{\nu}$ and any vector $w=\left(w_{1}, \ldots, w_{N+1}\right) \neq 0$, we have

$$
\text { (7) } \begin{aligned}
\left.\frac{d \tilde{H}_{\nu}(z+\lambda w)}{d \lambda}\right|_{\lambda=0}= & \int_{\partial \Delta_{\nu} \cap S_{z^{0} \cdot \sigma_{1}}} \frac{f(\zeta) \sum_{j=1}^{N+1}(\partial / \partial z,) K(\zeta, z) w_{j}}{\Phi(\zeta, z)^{N+1}} \\
& -\int_{\partial \Delta_{\nu} \cap S_{z^{0} o_{1}}} \frac{N \sum_{j=1}^{N+1}\left(\partial \Phi(\zeta, z) / \partial z_{j}\right) w_{j} K(\zeta, z)}{\Phi(\zeta, z)^{N+1}} .
\end{aligned}
$$


LEMMA 3. Let $f(\zeta) \in H^{\infty}(\Delta)$. Then for any point $z^{0} \in \partial \Delta$ and any point $z \in \partial\left(S_{z, 0} \cap D_{\nu}\right) \mid \partial \Delta_{\nu}\left(\sigma<\left(\sigma_{3} / 2\right)\right)$, we have

$$
\left|\frac{d \tilde{H}_{\nu}\left(\zeta^{0}+\lambda\left(z-\zeta^{0}\right)\right)}{d \lambda}\right|_{\lambda=1}\left|\leq \gamma_{5} \sup _{\zeta \in \Delta}\right| f(\zeta) \mid,
$$

where $\zeta^{0}=\zeta^{0}(z)$ and $\gamma_{5}$ depends only on $D$ and $\Delta$.

Proof. We set $\varepsilon=\left|z_{N+1}\right|$, where

$$
z=\left(z_{1}, \ldots, z_{N+1}\right) \in \partial\left(S_{z^{0, \sigma}} \cap D_{\nu}\right) \mid \partial \Delta_{\nu} .
$$

Then Lemma 2 implies the inequalities

$$
\varepsilon \leq\left|\zeta^{0}-z\right| \leq\left\{\frac{\rho(z)-\rho\left(\zeta^{0}\right)}{\gamma_{3}}\right\}^{1 / 2} \leq \frac{\varepsilon}{\left(\gamma_{3} \gamma_{4}\right) 1 / 2} .
$$

Since $\Sigma_{i=1}^{N+1}\left(\partial \rho / \partial \zeta_{i}\right)\left(\zeta^{0}\right)\left(\zeta_{i}^{0}-z_{i}\right)=0$, it follows that

$$
\begin{aligned}
\left|\sum_{i=1}^{N+1} \frac{\partial \Phi}{\partial z_{i}}(\zeta, z)\left(\zeta_{l}^{0}-z_{i}\right)\right|=\left|\sum_{i=1}^{N+1}\left(\frac{\partial \Phi(\zeta, z)}{\partial z_{i}}+2 \frac{\partial \rho}{\partial \zeta_{i}}\left(\zeta^{0}\right)\right)\left(\zeta_{i}^{0}-z_{i}\right)\right| \\
\quad \leq\left|\sum_{i=1}^{N+1}\left(\frac{\partial \Phi}{\partial z_{i}}(\zeta, z)-\frac{\partial \Phi}{\partial z_{i}}\left(\zeta^{0}, z\right)+O\left(\left|\zeta^{0}-z\right|\right)\right)\left(\zeta_{i}^{0}-z_{\imath}\right)\right| \\
\leq \gamma_{6} \varepsilon(|\zeta-z|+\varepsilon) .
\end{aligned}
$$

Here we have used the equation

$$
\frac{\partial \Phi}{\partial z_{i}}\left(\zeta^{0}, z\right)=-2 \frac{\partial \rho}{\partial \zeta_{i}}\left(\zeta^{0}\right)+O\left(\left|\zeta^{0}-z\right|\right) .
$$

By (7), we have

$$
\begin{aligned}
& \left.\left|\frac{d \tilde{H}_{\nu}\left(\zeta^{0}+\lambda\left(z-\zeta^{0}\right)\right)}{d \lambda}\right|_{\lambda=1}|=| \frac{d \tilde{H}_{\nu}\left(z+\lambda\left(z-\zeta^{0}\right)\right)}{d \lambda}\right|_{\lambda=0} \mid \\
& \quad \leq \gamma_{7} \int_{\partial \Delta_{\nu} \cap S_{z^{0 . \sigma_{1}}}} \frac{|f(\zeta)|\left|z-\zeta^{0}\right|}{|\Phi(\zeta, z)|^{N+1}} d \lambda+\gamma_{8} \int_{\partial \Delta_{\nu} \cap S_{z^{0 . \sigma_{1}}}} \frac{|f(\zeta)| \varepsilon(|\zeta-z|+\varepsilon)}{|\Phi(\zeta, z)|^{N+1}} d \lambda .
\end{aligned}
$$

We can choose coordinates $\left(\eta_{1}(\zeta), \ldots, \eta_{N+1}(\zeta)\right)$ in $S_{z^{0, \sigma_{3}}}$ such that $\eta_{1}(\zeta)=$ $\rho(\zeta)-\rho(z)+i \operatorname{Im} \Phi(\zeta, z)$. Then

$$
|\Phi(\zeta, z)| \geq \gamma_{9}\left[\left(t_{1}+|\zeta-z|^{2}\right)^{2}+t_{2}^{2}\right]^{1 / 2}
$$


and

$$
|\zeta-z| \geq \gamma_{10}\left(t_{1}^{2}+\cdots+t_{2 N}^{2}+\varepsilon^{2}\right)^{1 / 2} \geq \gamma_{11}|\zeta-z|,
$$

where we have written $\eta_{i}(\zeta)=t_{2 i-1}+\sqrt{-1} t_{2 i}(i=1,2, \ldots, N+1)$. Then we have

$$
\begin{aligned}
& \left|\frac{d F\left(\zeta^{0}+\lambda\left(z-\zeta^{0}\right)\right)}{d \lambda}\right|_{\lambda=1}\left|\leq \gamma_{12} \sup _{\zeta \in \Delta}\right| f(\zeta) \mid \\
& \times\left\{\varepsilon \int_{\substack{t_{2}^{2}+\cdots+t_{2 N}^{2} \leq 1 \\
t_{1} \geq 0}} \frac{d t_{2} d t_{3} \cdots d t_{2 N}}{\left[\left(t_{1}+t_{2}^{2}+\cdots+t_{2 N}^{2}+\varepsilon^{2}\right)^{2}+t_{2}^{2}\right]^{N / 2}}\right. \\
& +\varepsilon \int_{\substack{t_{2}^{2}+\cdots+t_{2 N}^{2} \leq 1 \\
t_{1} \geq 0}} \frac{\left(t_{1}^{2}+t_{2}^{2}+\cdots+t_{2 N}^{2}+\varepsilon^{2}\right)^{1 / 2} d t_{2} \cdots d t_{2 N}}{\left[\left(t_{1}+t_{2}^{2}+\cdots t_{2 N}^{2}+\varepsilon^{2}\right)^{2}+t_{2}^{2}\right]^{N+1 / 2}} \\
& \left.+\varepsilon^{2} \int_{\substack{t_{2}^{2}+\cdots+t_{2 N}^{2} \leq 1 \\
t_{1} \geq 0}} \frac{d t_{2} \cdots d t_{2 N}}{\left[\left(t_{1}+t_{2}^{2}+\cdots+t_{2 N}^{2}+\varepsilon^{2}\right)^{2}+t_{2}^{2}\right]^{N+1 / 2}}\right\}
\end{aligned}
$$

(by G. M. Henkin [5])

$$
\leq \gamma_{13} \sup _{\zeta \in \Delta}|f(\zeta)|
$$

We want to have

$$
\sup _{z \in D_{\nu}}\left|H_{\nu}(z)\right| \leq \gamma_{14} \sup _{\zeta \in \Delta}|f(\zeta)|
$$

where $\gamma_{14}$ depends only on $D$ and $\Delta$. We shall denote by $\left(\partial \Delta_{\nu}\right)_{\sigma}$ the $\sigma$-neighborhood of $\partial \Delta_{\nu}$. Since the function $H_{\nu}(z)$ is holomorphic at all points $z \in \bar{D}_{\nu} \mid \partial \Delta_{\nu}$, we have

$$
\sup _{z \in D_{\nu}}\left|H_{\nu}(z)\right| \leq \sup _{z \in \partial D_{\nu}\left(\partial \Delta_{\nu}\right)_{\sigma}}\left|H_{\nu}(z)\right|+\sup _{z \in\left[\left(\partial \Delta_{\nu}\right)_{\sigma} \mid \partial \Delta_{\nu}\right] \cap \partial D_{\nu}}\left|H_{\nu}(z)\right| .
$$

We obtain

$$
\begin{aligned}
& \sup _{z \in \partial D_{\nu}\left(\partial \Delta_{\nu}\right)_{\sigma}}\left|H_{\nu}(z)\right| \\
& \quad \leq \gamma_{15}\left[\int_{t_{2}^{2}+\cdots t_{2 N}^{2} \leq 1} \frac{d t_{2} \cdots d t_{2 N}}{\left[\left(t_{2}^{2}+\cdots t_{2 N}^{2}+\sigma^{2}\right)^{2}+t_{2}^{2}\right]^{N / 2}}\right] \sup _{\zeta \in \Delta}|f(\zeta)| \\
& \quad \leq \gamma_{16} \sup _{\zeta \in \Delta}|f(\zeta)| .
\end{aligned}
$$


Let $\sigma<16 \sigma_{3}$. We now fix $z \in\left[\left(\partial \Delta_{\nu}\right)_{\sigma}-\partial \Delta_{\nu}\right] \cap \partial D_{\nu}$. We take $\nu$ so large that one can find $z^{0} \in \partial \Delta$ such that $z \in S_{z^{0,2 \sigma}}$. Then by Lemma 2, there exists a solution $\zeta^{0}=\zeta^{0}(z)$ of system (2) belonging to the set $S_{z^{0,4 \sigma}} \cap \tilde{\Delta}$ and satisfying the inequalities

$$
\gamma_{3}\left|z_{N+1}\right|^{2} \leq \rho(z)-\rho\left(\zeta^{0}\right) \leq\left|z_{N+1}\right|^{2} / \gamma_{4} .
$$

Let $T_{\nu}=\left\{\lambda \in C: z(\lambda)=\zeta^{0}+\lambda\left(z-\zeta^{0}\right) \in D_{\nu} \cap S_{z^{0,4 \sigma}}\right\}$. $T_{\nu}$ is a convex domain containing $\lambda=0$. For any $\lambda$ we have

$$
\sum_{i=1}^{N+1} \frac{\partial \rho}{\partial \zeta_{i}}\left(\zeta^{0}\right)\left(\zeta_{i}^{0}-z_{i}(\lambda)\right)=0
$$

From this we have

$$
\left|z(\lambda)-\zeta^{0}\right|^{2} \leq \frac{1}{\gamma_{17}}\left\{\rho(z(\lambda))-\rho\left(\zeta^{0}\right)\right\}
$$

Hence for $\lambda \in \partial T_{\nu}$ with $z(\lambda) \in \partial D_{\nu}$, we obtain

$$
\begin{aligned}
\left|z(\lambda)-z^{0}\right| & \leq\left|z(\lambda)-\zeta^{0}\right|+\left|\zeta^{0}-z^{0}\right| \\
& \leq \frac{1}{\sqrt{\gamma_{17}}}\left(\rho(z(\lambda))-\rho\left(\zeta^{0}\right)\right)^{1 / 2}+\frac{\sigma_{3}}{4} \\
& =\frac{1}{\sqrt{\gamma_{17}}}\left(\rho(z)-\rho\left(\zeta^{0}\right)\right)^{1 / 2}+\frac{\sigma_{3}}{4} \\
& \leq \frac{\varepsilon}{\sqrt{\gamma_{4} \gamma_{17}}}+\frac{\sigma_{3}}{4} \leq \frac{\sigma}{\sqrt{\gamma_{4} \gamma_{17}}}+\frac{\sigma_{3}}{4} .
\end{aligned}
$$

We impose the further restriction that the constant $\sigma<\sigma_{3} \sqrt{\gamma_{4} \gamma_{17}} / 4$. Then $\left|z(\lambda)-z^{0}\right|<\sigma_{3} / 2$. Therefore $z(\lambda) \in S_{z^{0, \sigma_{3} / 2}}$. Since the point $\zeta^{0}(z)$ satisfies system (2) with any $z(\lambda)$ satisfying $\lambda \in \partial T_{\nu}$ and $z(\lambda) \in \partial D_{\nu}$, it follows that $\zeta^{0}(z(\lambda))=\zeta^{0}(z)$ for any $\lambda \in \partial T_{\nu}$ with $z(\lambda) \in \partial D_{\nu}$. Moreover

$$
\begin{aligned}
\frac{|\lambda| \varepsilon}{\gamma_{3} \gamma_{4}} & \geq \frac{|\lambda|}{\gamma_{3}}\left(\rho(z)-\rho\left(\zeta^{0}\right)\right) \geq|\lambda|\left|z-\zeta^{0}\right|=\left|z(\lambda)-\zeta^{0}\right| \\
& \geq\left(\gamma_{4}\left(\rho(z(\lambda))-\rho\left(\zeta^{0}\right)\right)\right)^{1 / 2} \\
& =\left[\gamma_{4}\left(\rho(z)-\rho\left(\zeta^{0}\right)\right)\right]^{1 / 2} \geq\left(\gamma_{3} \gamma_{4}\right)^{1 / 2} \varepsilon .
\end{aligned}
$$

Therefore $|\lambda| \geq \gamma_{3} \gamma_{4}$ for any $\lambda \in \partial T_{\nu}$ with $z(\lambda) \in \partial D_{\nu}$. If $\lambda \in \partial T_{\nu}$ and $z(\lambda) \in S_{z^{0.4 \sigma}}$, there exists $\gamma_{18}>0$ such that $|\lambda| \geq \gamma_{18}$. Let $\gamma_{19}=$ $\min \left(\gamma_{3} \gamma_{4}, \gamma_{18}\right)$. Then

$$
|\lambda| \geq \gamma_{19} \text { for any } \lambda \in \partial T_{\nu}
$$


By Lemma 3, we have

$$
\left|\frac{d H_{\nu}\left(\zeta^{0}+t\left(z(\lambda)-\zeta^{0}\right)\right)}{d t}\right|_{t=1}\left|\leq \gamma_{5} \sup _{\zeta \in \Delta}\right| f(\zeta) \mid
$$

for any $\lambda \in \partial T_{\nu}$. We note that

$$
\left.\frac{d H_{\nu}\left(\zeta^{0}+t\left(z(\lambda)-\zeta^{0}\right)\right)}{d t}\right|_{t=1}=\frac{d H_{\nu}\left(\zeta^{0}+\lambda\left(z-\zeta^{0}\right)\right)}{d \lambda} .
$$

From (8), (9) and (10), we have

$$
\left|\frac{d H_{\nu}\left(\zeta^{0}+\lambda\left(z-\zeta^{0}\right)\right)}{d \lambda}\right| \leq \frac{\gamma_{5}}{|\lambda|} \sup _{\zeta \in \Delta}|f(\zeta)| \leq \frac{\gamma_{5}}{\gamma_{19}} \sup _{\zeta \in \Delta}|f(\zeta)|
$$

for any $\lambda \in \partial T_{\nu}$. Since the function $d H_{\nu}\left(\zeta^{0}+\lambda\left(z-\zeta^{0}\right)\right) / d \lambda$ is holomorphic in $\lambda$ for all $\lambda \in \bar{T}_{\nu}$, it follows that

$$
\sup _{\lambda \in T_{\nu}}\left|\frac{d H_{\nu}\left(\zeta^{0}+\lambda\left(z-\zeta^{0}\right)\right)}{d \lambda}\right| \leq \frac{\gamma_{5}}{\gamma_{19}} \sup _{\zeta \in \Delta}|f(\zeta)| .
$$

Consequently

$$
\left|H_{\nu}(z)-H_{\nu}\left(\zeta^{0}\right)\right|=\left|\int_{0}^{1} \frac{d}{d \lambda} H_{\nu}\left(\zeta^{0}+\lambda\left(z-\zeta^{0}\right)\right) d \lambda\right| \leq \frac{\gamma_{5}}{\gamma_{19}} \sup _{\zeta \in \Delta}|f(\zeta)| .
$$

From (8), $\zeta^{0} \in \Delta_{\nu}$. Since $H_{\nu}\left(\zeta^{0}\right)=f\left(\zeta^{0}\right)$, we have

$$
\left|H_{\nu}(z)\right| \leq\left(\frac{\gamma_{5}}{\gamma_{19}}+1\right) \sup _{\zeta \in \Delta}|f(\zeta)| \text {. }
$$

Therefore

$$
\sup _{z \in D_{\nu}}\left|H_{\nu}(z)\right| \leq \gamma_{20} \sup _{\zeta \in \Delta}|f(\zeta)|
$$

Hence

$$
\sup _{z \in D}|H(z)| \leq \gamma_{20} \sup _{\zeta \in \Delta}|f(\zeta)| .
$$

The next step is to show that if $f \in A(\Delta)$, then also $H(z)=L(f)(z)$ $\in A(D)$. In this case we have assumed that $\Delta$ has no singular points. Therefore by N. Kerzman [6], there exists a sequence $\left\{f_{k}\right\}_{k=1}^{\infty}$ of functions holomorphic in a neighborhood of $\bar{\Delta}$ in $\tilde{\Delta}$ such that $\left\|f_{k}-f\right\|_{\Delta} \rightarrow 0$ when $k \rightarrow \infty$. By the continuity of $L$ it suffices to prove that each $L f_{k}$ is in $A(D)$. Hence we can suppose $f$ is holomorphic in $\bar{\Delta}^{\prime}\left(\bar{\Delta} \subset \Delta^{\prime} \subset \bar{\Delta} \subset \tilde{\Delta}\right)$. 
Let $z^{0} \in \partial \Delta$ and let $z \in S_{z^{0, \sigma / 2}} \cap\left(\bar{D}_{\nu} \mid \partial \Delta_{\nu}\right)$. By Stokes' formula, we have

$$
\begin{aligned}
H_{\nu}(z)= & \int_{\partial \Delta_{\nu}} \frac{f(\zeta) K(\zeta, z)}{\Phi(\zeta, z)^{N}} \\
= & \int_{\partial \Delta^{\prime}} \frac{f(\zeta) K(\zeta, z)}{\Phi(\zeta, z)^{N}}-\int_{\Delta^{\prime}-\Delta_{\nu}} f(\zeta) \bar{\partial}_{\zeta}\left(\frac{K(\zeta, z)}{\Phi(\zeta, z)^{N}}\right) \\
= & \int_{\partial \Delta^{\prime}} \frac{f(\zeta) K(\zeta, z)}{\Phi(\zeta, z)^{N}}-\int_{\left(\Delta^{\prime}-\Delta_{\nu}\right) \cap S_{z_{0}^{0.2 \sigma}}} f(\zeta) \bar{\partial}_{\zeta}\left(\frac{K(\zeta, z)}{\Phi(\zeta, z)^{N}}\right) \\
& -\int_{\left(\Delta^{\prime}-\Delta_{\nu}\right) \mid S_{z^{0.2 \sigma}}} f(\zeta) \bar{\partial}_{\zeta}\left(\frac{K(\zeta, z)}{\Phi(\zeta, z)^{N}}\right) .
\end{aligned}
$$

The first and the third term on the left are continuous in $z^{0}$. Therefore it is sufficient to show that, if we set

$$
F_{\nu}(z)=\int_{\left(\Delta^{\prime}-\Delta_{\nu}\right) \cap S_{z} 0.2 \sigma} f(\zeta) \bar{\partial}_{\zeta}\left(\frac{K(\zeta, z)}{\Phi(\zeta, z)^{N}}\right),
$$

then $F_{\nu}(z)$ is continuous at $z^{0}$.

LEMMA 4. Let $z \in S_{z^{0,0 / 2}} \cap\left(\bar{D}_{\nu} \mid \partial \Delta\right)$. Then

$$
\left|\frac{d F_{\nu}\left(\zeta^{0}+\lambda\left(z-\zeta^{0}\right)\right)}{d \lambda}\right|_{\lambda=1}\left|\leq \gamma_{21} \varepsilon\right| \log \varepsilon\left|\sup _{\zeta \in \tilde{\Delta}}\right| f(\zeta) \mid .
$$

Proof. We can write

$$
\begin{aligned}
F_{\nu}(z)= & \int_{\left(\Delta^{\prime}-\Delta_{\nu}\right) \cap S_{z^{0.2 \sigma}}} f(\zeta) \frac{A(\zeta, z)}{\Phi(\zeta, z)^{N}} \\
& +\int_{\left(\Delta^{\prime}-\Delta_{\nu}\right) \cap S_{z^{0.2 \sigma}}} \frac{f(\zeta) \Sigma_{j=1}^{N+1}\left(\zeta_{j}-z_{j}\right) B_{j}(\zeta, z)}{\Phi(\zeta, z)^{N+1}}
\end{aligned}
$$

where $A(\zeta, z)$ and $B_{j}(\zeta, z)$ are $(N, N)$ forms which are continuous in $\zeta$ and holomorphic in $z$. Therefore

$$
\begin{aligned}
\left|\frac{d F_{\nu}\left(\zeta^{0}+\lambda\left(z-\zeta^{0}\right)\right)}{d \lambda}\right|_{\lambda=1} \mid \leq & \gamma_{22} \int_{\left(\Delta^{\prime}-\Delta_{\nu}\right) \cap S_{z^{0.2 \sigma}}} \frac{\varepsilon}{\Phi(\zeta, z)^{N+1}} d \lambda \\
& +\gamma_{23} \int_{\left(\Delta^{\prime}-\Delta_{\nu}\right) \cap S_{z^{0}, 2 \sigma}} \frac{|\zeta-z| \varepsilon(|\zeta-z|+\varepsilon)}{|\Phi(\zeta, z)|^{N+2}} d \lambda
\end{aligned}
$$


(by the estimates of G. M. Henkin [5])

$$
\leq \gamma_{24} \varepsilon|\log \varepsilon| \sup _{\zeta \in \tilde{\Delta}}|f(\zeta)| .
$$

Therefore Lemma 4 is proved.

Using the method of Henkin [5], we have

$$
\left|F_{\nu}(z)-F_{\nu}\left(z^{0}\right)\right| \leq \gamma_{25} \sigma|\log \sigma| \sup _{\zeta \in \Delta^{\prime}}|f(\zeta)|+\gamma_{26} \sigma \sup _{\zeta \in \Delta^{\prime}}|\operatorname{grad} f(\zeta)| .
$$

Therefore $F_{\nu}(z)$ is continuous at $z^{0}$. Therefore the theorem is proved.

\section{REFERENCES}

[1] K. Adachi, Extending bounded holomorphic functions from certain subvarieties of a strongly pseudoconvex domain, Bull. Fac. Sci., Ibaraki Univ., Math., No. 8, (1976), $1-7$.

[2] E. Amar, $\bar{\partial}-$ Cohomologie $C^{\infty}$ et applications, preprint.

[3] F. Beatrous, Jr., Hölder estimates for the $\bar{\partial}$ equation with a support condition, Pacific J. Math., 90, No. 2, (1980), 249-257.

[4] J. E. Fornaess, Embedding strictly pseudoconvex domains in convex domains, Amer. J. Math., 98 (1976), 529-569.

[5] G. M. Henkin, Continuation of bounded holomorphic functions from submanifolds in general position to strictly pseudoconvex domains, Izv. Akad. Nauk SSSR, 36 (1972), 540-567. (English translation: Math. USSR Izvestija, 6 (1972), 536-563.)

[6] N. Kerzman, Hölder and $L^{p}$-estimates for solutions of $\bar{\partial} u=f$ in strongly pseudoconvex domains, Comm. Pure Appl. Math., 24 (1971), 301-380.

[7] R. M. Range, Holomorphic approximation near strictly pseudoconvex boundary points, Math. Ann., 201 (1973), 9-17.

[8] E. L. Stout, An integral formula for holomorphic functions on strictly pseudoconvex hypersurfaces, Duke Math. J., 42, No. 2 (1975), 347-356.

Received November 19, 1981 and in revised form May 26, 1982.

NAGASAKI UNIVERSITY

NAGASAKI 852, JAPAN 



\section{PACIFIC JOURNAL OF MATHEMATICS \\ EDITORS}

DONALD BABBITT (Managing Editor)

University of California

Los Angeles, CA 90024

Hugo RossI

University of Utah

Salt Lake City, UT 84112

C. C. MOOre and ARThur OGus

University of California

Berkeley, CA 94720
J. Dugundi

Department of Mathematics

University of Southern California

Los Angeles, CA 90089-1113

R. FINN and H. SAMELSON

Stanford University

Stanford, CA 94305

ASSOCIATE EDITORS

$\begin{array}{lllll}\text { R. Arens } & \text { E. F. Beckenbach } & \text { B. H. Neumann } & \text { F. Wolf } & \text { K. Yoshida }\end{array}$ $(1906-1982)$

\section{SUPPORTING INSTITUTIONS}

UNIVERSITY OF ARIZONA

UNIVERSITY OF BRITISH COLUMBIA

CALIFORNIA INSTITUTE OF TECHNOLOGY

UNIVERSITY OF CALIFORNIA

MONTANA STATE UNIVERSITY

UNIVERSITY OF NEVADA, RENO

NEW MEXICO STATE UNIVERSITY

OREGON STATE UNIVERSITY
UNIVERSITY OF OREGON

UNIVERSITY OF SOUTHERN CALIFORNIA

STANFORD UNIVERSITY

UNIVERSITY OF HAWAII

UNIVERSITY OF TOKYO

UNIVERSITY OF UTAH

WASHINGTON STATE UNIVERSITY

UNIVERSITY OF WASHINGTON 


\section{Pacific Journal of Mathematics}

\section{Vol. 110, No. $1 \quad$ September, 1984}

Wojciech Abramczuk, A class of surjective convolution operators $\ldots \ldots \ldots \ldots 1$

K. Adachi, Extending bounded holomorphic functions from certain

subvarieties of a weakly pseudoconvex domain $\ldots \ldots \ldots \ldots \ldots \ldots$

Malvina Florica Baica, An algorithm in a complex field and its application

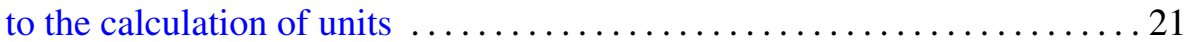

Giuliana Bianchi and Robert Cori, Colorings of hypermaps and a

conjecture of Brenner and Lyndon $\ldots \ldots \ldots \ldots \ldots \ldots \ldots \ldots \ldots \ldots \ldots \ldots$

Ronald James Evans, Determinations of Jacobsthal sums . . .......... 49

Leslie Foged, Characterizations of $\aleph$-spaces .................... 59

Nassif A. Ghoussoub and Paulette Saab, Weak compactness in spaces of

Bochner integrable functions and the Radon-Nikodým property . . . . . . 65

J. Gómez Gil, On local convexity of bounded weak topologies on Banach

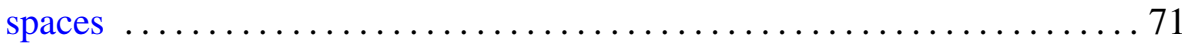

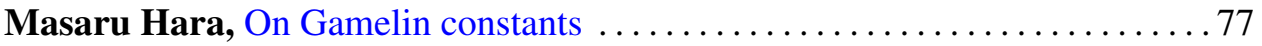

Wilfried Hauenschild, Eberhard Kaniuth and Ajay Kumar, Harmonic

analysis on central hypergroups and induced representations $\ldots \ldots \ldots 83$

Eugenio Hernandez, An interpolation theorem for analytic families of

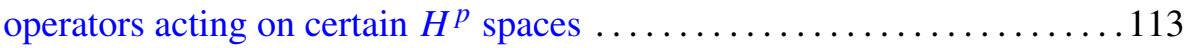

Thomas Alan Keagy, On "Tauberian theorems via block-dominated

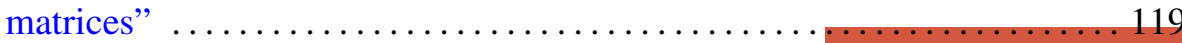

Thomas Landes, Permanence properties of normal structure $\ldots \ldots \ldots \ldots \ldots 125$

Daniel Henry Luecking, Closed ranged restriction operators on weighted

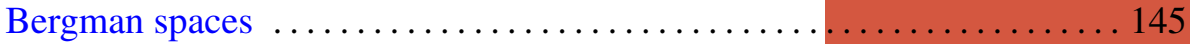

Albert Thomas Lundell, The $p$-equivalence of $\mathrm{SO}(2 n+1)$ and $\mathrm{Sp}(n) \ldots \ldots 161$

Mark D. Meyerson, Remarks on Fenn's "the table theorem" and Zaks' "the chair theorem" ..................................... 167

Marvin Victor Mielke, Homotopically trivial toposes . . . . . . . . . . 171

Gerard J. Murphy, Hyperinvariant subspaces and the topology on Lat A . . 183

Subhashis Nag, On the holomorphy of maps from a complex to a real manifold

Edgar Milan Palmer and Robert William Robinson, Enumeration of self-dual configurations ................................. 203

John J. Walsh and David Clifford Wilson, Continuous decompositions

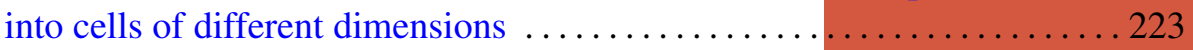

Walter John Whiteley, Infinitesimal motions of a bipartite framework .....233 